\title{
Electromagnetic Metrology for NEMS
}

\author{
Ling Hao*, John Gallop*, and Jie Chen ${ }^{\dagger}$ \\ * National Physical Laboratory \\ Hampton Road, Teddington, TW11 0LW, UK \\ E-mail: ling.hao@npl.co.uk \\ ${ }^{\dagger}$ Brunel University London, Uxbridge, UB8 3PH, UK
}

\begin{abstract}
This paper outlines how demands on electromagnetic metrology have developed over recent years with the continuing reduction in the typical length scale of electronic components and circuits. In addition novel materials, especially 2D self-supporting structures such as graphene, have properties quite unlike conventional systems. At the nanoscale radical changes occur in techniques required and even in the physical quantities measured. This paper focusses on the example of a nearfield scanning microwave microscope and its application to nanoelectromechanical system resonators to illustrate these changes and challenges.
\end{abstract}

Index Terms - nanoscale electromagnetic metrology, graphene, NEMS, microwave, NSMM.

\section{INTRODUCTION}

Immense strides have been taken over the past 50 years in replacing artefact definitions of the SI base units by those based on fundamental constants [1]. It is important to recognise that the development of metrology relating to the SI is not 'complete' once the base units are all defined in this way. New physics, new materials and new technologies will always emerge and will require developments and extensions to the SI. A current significant development involves the extension of precise metrology towards the nanoscale. Developments in this area do not only require novel techniques but it must also be acknowledged that as the length scale reduces the behavior of the system moves away from the classical concept of an ensemble of particles towards an approach based on a finite number of entities, whether they be molecules, atoms, photons, spins or even phonons. Ultimately metrology at the nanoscale is concerned with the measurement of single entities and the inevitable quantum mechanical issues which arise. However, long before the single entity level or true quantum mechanical behaviour is attained, many other challenges to classical electrical metrology arise as the length scale decreases [2]. Some key issues are outlined in the following sections.

\section{CONTACTS, INTERFACES \& DIMENSIONALITY}

Conventional electromagnetic measurements involve attaching conducting leads to a specimen under test (SUT) and then applying stationary or time varying currents or voltages to these leads while measuring the response of the SUT via other connections. Measurement of conductivity, resistance, capacitance, inductance and magnetization can all be carried out in this way for samples of millimetre size and larger, at relatively low frequencies (e.g. $<1 \mathrm{GHz}$ ).

With the development of micron scale lithography new techniques for electromagnetic metrology were needed. Increasingly non-linear properties of the SUT were required to be measured, due to increased focus on semiconductors. Further developments towards ever smaller lengths have led to, and enabled, nanoscale electromagnetic devices. Particularly important have been the improvements in electron beam lithography (EBL), focused ion beam (FIB) and scanned probe microscopies (SPM), including atomic force microscopes and scanning tunneling microscopes. Patterned devices on the scale down to a few tens of nanometers in all three dimensions are now possible, leading to increased focus on connections, interfaces and dimensionality of the SUT.

\section{ELECTRICAL MEASUREMENTS ON REDUCED DIMENSIONS}

The complexity of the requirements of nanoscale electromagnetic measurements is exemplified by the needs of the new two-dimensional material graphene. As the first selfsupporting single atomic layer material, graphene has high electrical, thermal conductivity and its interfaces with external circuits are problematic. In addition the conductivity and mobility of this zero band-gap semiconductor are both easily influenced by an electric field acting perpendicular to the graphene surface. Many techniques have been developed to deal with these issues but here we discuss one important example [3] which overcomes some of these difficulties, a nearfield scanning microwave microscope (NSMM).

\section{A. Scanning Microwave Microscope}

The paradoxical ability to use microwaves (typical free space wavelength $\sim 1 \mathrm{~cm}$ ) to image on the nanoscale requires the use of the strongly localized evanescent microwave nearfield existing in the neighbourhood of a sharply pointed conductor driven by a microwave signal. An additional advantage of using microwaves that a high $\mathrm{Q}$ resonator (unavailable at lower frequencies) can be incorporated with the sharp tip, to give enhanced gain. A home-built NPL NSMM (see Fig. 1) consists of a quarter wavelength coaxial resonator, loaded by a high permittivity $\left(\varepsilon_{r} \sim 20\right)$ dielectric material, with a resonant frequency of $4 \mathrm{GHz}$ in a length of only $4 \mathrm{~mm}$ and a 
radius of $1.25 \mathrm{~mm} \mathrm{[4].} \mathrm{with} \mathrm{an} \mathrm{unloaded} \mathrm{Q} \mathrm{value} \mathrm{of} \mathrm{700.} \mathrm{To} \mathrm{the}$ open end of the centre conductor is attached a short length of sharply tapered tungsten wire with an estimated radius of curvature at the tip of $\sim 1 \mu \mathrm{m}$. The microwave field from the cavity extends into this region and is highly enhanced at the tip due to the sharp radius of curvature. The near-field region represents an external contribution to the total stored energy in the coaxial resonator. If a conducting or dielectric NEMS resonator moves in this high field region the field distribution will be changed and as a result the resonator properties will be slightly altered. This response shifts both the resonant frequency and the $\mathrm{Q}$ of the resonator.

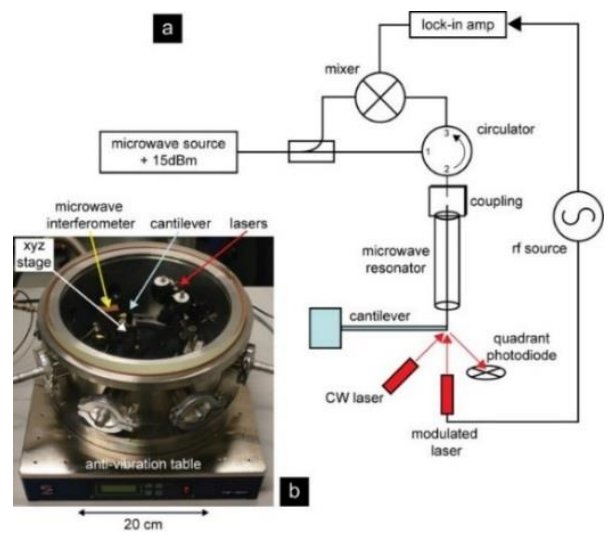

Fig. 1. a) Schematic of scanned microwave microscope circuit for homodyne detection of surface impedance b) Photo of scanning and readout system inside vacuum chamber with glass lid.

To interrogate the resonator a modified homodyne detection system is shown in Fig. 1. A synthesised microwave source with output power up to $+21 \mathrm{dBm}$ may be amplitude or frequency modulated by an external signal supplied by an r.f. source. The microwave output is fed to a splitter where a fraction of the power is tapped off. The remaining power is fed to a circulator with the dielectric coaxial resonator being connected to the adjacent port. The reflected signal from the resonator is returned to the r.f. input of a microwave mixer, with the tapped off component of the microwave power.

The system has been used to make 2D scans across both dielectric and conducting thin films and is able to resolve changes in the local properties down to a length scale of around $1 \mu \mathrm{m}$. We have recently used this to image variations in local sheet resistance of transferred CVD graphene films.

\section{B. NEMS Resonators}

The convolution of physical size, real and imaginary parts of electric conductivity and relative permeability of any SUT make accurate measurements of nanoscale electromagnetic properties complex. Additional complexity arises if nanoelectromechanical systems (NEMS) are also considered. For example the radiation pressure at the tip of the NSMM exerts a measurable force on a conducting sample and if this is free to move, this will change the reflected microwave signal from the probe, making it hard to distinguish a spatial from an electrical variation. However the interaction between tip and sample can be put to good use since by modulating the amplitude of the microwave signal at a variable frequency any mechanical resonances in the freely suspended sample may be detected. The force exerted by the microwave field on the mechanical resonator depends on the electric field strength since the near-field mechanical resonator combination can be treated as a variable capacitance. In order to apply a time varying force the amplitude of the microwave field must be modulated. In this way motion of micro and nanoscale cantilevers has been imaged, measuring the shape of the displacement along the diameter or length of a drum and a cantilever. The technique can detect non-linear behavior including Duffing type response and has been used to investigate graphene drum resonators.

\section{Traceability}

Current developments involve converting the scanned raw microwave resonance data to conductivity and permittivity of the underlying material. Simple circuit based modelling provides good measures of relative changes in both real and imaginary components of SUT impedance. However scaling these variations to material parameters such as conductivity or permittivity is more complex. Our approach uses the mean parameter values measured with a far field dielectric resonator. A second system employs the observed frequency shift in the microwave resonance as a servo signal to control the distance between tip and SUT. This has been used extensively [5] but is not reliable if the permittivity of the surface is also subject to spatial variation. A more precise approach, currently under test, uses standard thin film conducting and insulating samples.

\section{ACKNOWLEDGEMENT}

This work is funded by the UK NMS Program and EPSRC.

\section{REFERENCES}

[1] Martin J T Milton, Richard Davis and Nick Fletcher, 'Towards a new SI: a review of progress made since 2011', Metrologia vol. 51, R21-R30, 2014.

[2] J C Gallop, 'Fundamental metrology in the future: Measuring the single quantum', Eu. Phys. Jnl., vol. 172, pp399-408, 2009.

[3] Atif Imtiaz, Thomas Mitchell Wallis, and Pavel Kabos, 'NearField Scanning Microwave Microscopy' IEEE Microwave Magazine · January 2014 pp52-64.

[4] Ling Hao, Stefan Goniszewski, John Gallop and Jie Chen, 'Development of Near-field Microwave Methods for NEMS Resonators', IEEE International Conference on Nanotechnology 2013, pp.379-383.

[5] S. E. de Graaf, A. V. Danilov, A. Adamyan, and S. E. Kubatkin, 'Tip-sample distance control for near-field scanning microwave microscopes,' Rev. Sci. Instrum, vol. 84, 023706, 2013. 